\title{
The management of malnutrition in hospital
}

\author{
BY SIMON P. ALLISON \\ Nottingham University Hospital, Nottingham NG7 $2 U H$
}

\begin{abstract}
You are not to permit your patient to encounter the terrible consequences of starvation because he does not ask for nutriment. Gentlemen, these results are due to good feeding. When I am gone, you may be at a loss for an epitaph for me. I give it to you in these words. He fed fevers.
\end{abstract}

ROBERT GRAVES

Dublin 1843

The management of malnutrition in hospital depends not only on a specialized nutrition team to provide the more sophisticated forms of artificial nutritional support, but on a number of other important conditions (Lennard-Jones, 1992). The first essential is a hospital management policy that nutrition is an important component of the quality of patient care, and that the appropriate resources will be made available. The second condition is an improvement in undergraduate and postgraduate training of medical and nursing staff, to enable them to understand the basics of clinical nutrition (Lennard-Jones et al. 1995). They need to appreciate that, assuming a normal previous weight, a loss of between 10 and $20 \%$ of body weight in association with illness causes significant deterioration in function which can slow recovery from illness or threaten life (Allison, 1992; Hill, 1992). They should know that muscle (Russell et al. 1983), respiratory (Arora \& Rochester, 1982) and thermoregulatory function (Mansell et al. 1990) are impaired, that immune responses and the resistance to infection are lower (Chandra, 1988), that wound healing is prevented (Windsor et al. 1988), and that with more severe weight loss both cardiovascular and gastrointestinal functions are impaired (Winick, 1979). Patients also become depressed and apathetic (Brozek, 1990) and may even be described as 'difficult'. Hospital stay is also prolonged by undernutrition (Robinson et al. 1987; Reilly et al. 1988) and may be shortened by treatment (Bastow et al. 1983; Rana et al. 1992). They should be aware that a substantial number of prospective controlled trials have shown benefit of nutritional support in undernourished patients, whether using oral supplements (Delmi et al. 1990; Larsson et al. 1990; Rana et al. 1992), or artificial nutrition via the enteral or parenteral route (Bastow et al. 1983; Veterans' Affairs Total Parenteral Nutrition Study Group, 1991; Von Meyenfeldt et al. 1992). Staff must be trained in the identification of malnutrition and in clinical decision making. Anorexia and weight loss are inevitable accompaniments of terminal illness, whether it be cancer, heart failure, respiratory failure or simply old age itself. In such instances, intervention may be merely officious and unkind. On the other hand, in the presence of recoverable disease, or where significant palliation can be achieved by nutritional intervention, this must be conducted in the simplest and most effective manner possible.

Assuming that the previously described conditions prevail, the next step is to design a screening system which is sufficiently simple and rapid that it can be carried out routinely for every admission by busy nursing and medical staff. The system should be sufficiently sensitive and specific to identify malnourished patients and those who are at risk of 
becoming malnourished during a prolonged hospital stay. It should indicate also the appropriate course of action, whether it be a referral to a dietitian, to the nutrition team, or even no action at all. It has already been established how very bad we are in hospitals at identifying patients at risk (McWhirter \& Pennington, 1994) and the options for screening have been discussed (Reilly, 1996). We have tried an assessment form which categorizes patients into broad groups of normality, and moderate or severe risk, but find that our nurses prefer a scoring system and, therefore, have adopted this approach.

With all these conditions in place, and having identified the patients at risk, how should we manage the problem?

\section{THE HIERARCHY OF DECISION MAKING}

Most patients at risk can be managed by simple means such as nursing help with meals, the ordering of special food, or the provision of supplements. Such measures can be taken by the nursing staff concerned. Persistent or more difficult problems should be referred by the nursing or medical staff to the dietitian, who should be established as an important member of the ward team. The dietitian should make a careful assessment of the patient and decide whether adequate nutrition can be given by mouth or by nasogastric tube. Last, in a minority of patients, the nutrition team (clinician, nutrition nurse, dietitian and pharmacist) may be called upon to give advice and carry out enteral nutrition (using naso-enteral, gastrostomy or jejunostomy) or, in cases of gastrointestinal failure, parenteral nutrition (via peripheral or central veins).

\section{HOSPITAL CATERING}

The nutritional care of patients has suffered from the separation of budgets into hotel and treatment costs. Catering has become the poor relation of management, and conceptual and budgetary barriers have divided it from dietetics. Catering services are run on a shoe-string and designed to cope with institutional meals, on the same model as student hostels or prisons. They are not targeted towards the sick. The problems lie not just in the purchase and preparation of food, but in its transport, delivery and presentation to the patient in a manner which will ensure its consumption. Most studies of the quality of hospital food are based on questionnaires which address taste or patient satisfaction rather than nutritional and dietary adequacy. To improve matters, conceptual barriers need to be swept away, and fundamental questions asked. Should the management of catering come under dietetic supervision, so that it can be targeted to patient groups, from those with obesity, diabetes and heart disease who need a healthy-eating programme, to the undernourished, in whom such considerations are irrelevant and where the administration of fat may be an important means of raising energy intake to adequate levels? Should hospitals disinvest in food preparation and buy in cooked-chilled food, reinvesting in quality control and delivery of food to the patients? This would allow targeting of menus to particular groups such as the long-stay elderly, in whom tastes and appetite may be very different from the fit, young orthopaedic patients. Such a policy has been developed at the Rikshospitalet in Copenhagen (J. Kondrup, personal communication), where there is a high-quality cooked-chilled menu. There is also a nutritional screening scheme in place, which results in the referral of 2000 patients per year to the Nutrition Department, which is run by a 
doctor and staffed by dietitians. The majority of these patients are well managed by highquality special diets, rich in energy and protein. Less than $10 \%$ of the patients so referred require artificial nutrition, enterally or parenterally. Because of the high quality of the food provided, there is less use of proprietary oral supplements, although these may have their important place. A recent study by Ödlund Olin et al. (1994) from Stockholm also showed that the provision of energy-dense hospital meals to elderly patients produced a $16 \%$ increase in energy intake and a 5\% increase in weight over 3 weeks, with improved mobility. This was at a cost increase of only $4 \%$.

We are currently engaged in a study of the role of hospital catering in the care of patients and the effect of nutritional policies on food waste and on hospital stay. We are also examining the effects of interventions such as extra snacks for the elderly, near-ward cooking facilities for particular patients, and the serving and presentation of meals by staff. In order to measure waste in a scientific manner, we have been trying to relate it to the amount of food provided. Rather than assuming that the portions provided meet the agreed standards, we have measured the content of the food and found an extremely wide range, with a mean of $60-70 \%$ of the amount assumed. Taking into account the number of meals missed through investigations and procedures, it can readily be calculated how much the patients' intake falls short of estimated requirements. It is no surprise, therefore, that patients lose weight and become undernourished during their hospital stay. The UK Department of Health and Nutrition Task Force Hospital Catering Project Team (1995) has just produced a report, Nutrition Guidelines for Hospital Catering under the Health of the Nation programme. It addresses many of these issues and, it is hoped, will result in some improvement.

Although Florence Nightingale in 1859 laid stress on nutrition as an important component of nursing care, the serving of meals has been relegated to untrained orderlies, who may not have an understanding of the feeding difficulties of particular patients. Perhaps we need a new grade of ward hostesses, who can be trained to ensure that the food delivered to the ward is presented to the patient in a way which ensures its consumption. We should also re-examine the role of near-ward kitchens to provide a service to patients with special needs. Our own experience of using a metabolic ward kitchen and cook in this way suggests that $10-15 \%$ of patients might benefit from this approach.

\section{THE ANORECTIC PATIENT}

Even when the quality and presentation of hospital food is optimal, there remain patients who are reluctant to eat, although they retain gastrointestinal function. As in all clinical situations, nutritional management must be seen as part of overall management. It is not sufficient to deliver proprietary supplements to the ward and hope for the best. When a patient, who has been screened and found to be at risk, is referred for further advice, some form of artificial nutrition may prove necessary. On the other hand, a broad assessment may allow the problem to be resolved by simple means. The point is best illustrated by some brief case histories.

\section{Case 1}

A 45-year-old male doctor, $1.7 \mathrm{~m}$ in height and $70 \mathrm{~kg}$ in weight (BMI 24.2), developed pain in the right thigh, fever and malaise. After 1 month, osteomyelitis of the right femur was drained. The pus grew Streptococcus milleri and anaerobic streptococci on culture. 
Benzylpenicillin and Metronidazole were administered. After a further 1 month, he relapsed with a fever of $38^{\circ}$ and was transferred to Nottingham University Hospital. His weight at this time was $59.9 \mathrm{~kg}$ (with marked oedema present). Serum albumin was $29 \mathrm{~g} / \mathrm{l}$ and haemoglobin $97 \mathrm{~g} / \mathrm{l}$. A large abscess of the right thigh was drained. The patient remained severely anorectic and nauseated with an oral intake less than $2 \cdot 1 \mathrm{MJ} / \mathrm{d}$. After subsequent loss of his oedema, his weight fell to $54.25 \mathrm{~kg}$ (BMI 18.8), revealing a total loss of $22.5 \%$ of his original body weight. Such weight loss being associated with severe deficits of function, his doctors became concerned about his worsening nutritional state. An assessment by the nutrition team suggested that the cause of such a severe degree of anorexia lay in the drugs he was receiving. The Metronidazole, therefore, was stopped and the patient's appetite recovered. Within 1 week he was consuming over 10.5 MJ daily. His wound started to heal and within 1 month he had gained $6 \mathrm{~kg}$ of real tissue weight and was able to be discharged. This patient's severe weight loss was due to a combination of increased metabolic rate from his sepsis and the anorexia associated not only with his illness, but with the drugs used to treat it. The removal of the cause of his anorexia obviated the need for any form of artificial nutrition.

\section{Case 2}

A 72-year-old man, who weighed $71 \mathrm{~kg} 1$ year before admission, underwent surgery for resection of sigmoid colon and some ileum for carcinoma of the colon, leaving a colostomy in place. Ileus and subacute obstruction persisted post-operatively and, after $15 \mathrm{~d}$, he was referred to the nutrition team for parenteral nutrition. At this time he weighed $61 \mathrm{~kg}$ with oedema. His real tissue weight was therefore $58 \mathrm{~kg}$ with a height of $1.75 \mathrm{~m}$ (BMI 18.7, weight loss $18 \%$ ). Serum albumin was $22 \mathrm{~g} / \mathrm{l}$. A trial of oral intake was attempted and, within $3 \mathrm{~d}$, the patient was able to take $6.7 \mathrm{MJ}$ by mouth when special meals were prepared for him by the ward-kitchen cook. As this met his resting energy requirements, parenteral nutrition was not required.

\section{Case 3}

A 75-year-old man had lost $10 \%$ of his body weight pre-operatively. He underwent resection of his sigmoid colon for a carcinoma and suffered an anastomotic leak postoperatively, resulting in a colonic fistula. He became very depressed with persistent anorexia. All attempts to encourage him to eat more or to take proprietary supplements caused him distress. His wound broke down, his serum albumin concentration dropped to $22 \mathrm{~g} / 1$ and he became oedematous. He was referred to the nutrition team for intravenous feeding. However, overnight nasogastric tube feeding by slow pump administration to give initially 4.2 MJ, increasing over 1 week to $8.4 \mathrm{MJ}$ nightly, resulted not only in an improved mood, sense of well-being and rapid wound healing and closure of his fistula, but also in a disinhibition of his appetite so that his voluntary oral intake by day increased to the point where it was meeting all his requirements. After $10 \mathrm{~d}$ the nasogastric tube feeding could be withdrawn. This case illustrates the adage that, 'if the gastrointestinal tract works use it', and also shows that distal-bowel fistulas can be managed by enteral feeding. The disinhibition of appetite by overnight nasogastric feeding has been reported previously (Bastow et al. 1985), although its mechanism remains obscure. 


\section{Case 4}

A 73-year-old woman, $1.55 \mathrm{~m}$ in height and $35 \mathrm{~kg}$ in weight (BMI 13.8), and a remembered weight 2 years before of $51 \mathrm{~kg}$ (BMI 21.3), had lost 35\% of her body weight. She and her twin sister both developed thyrotoxicosis with its consequent increase in metabolic rate. However, unlike her sister, the patient developed severe depression and anorexia, resulting in cachexia. Despite adequate treatment of her thyrotoxicosis and depression, and the giving of special meals, her oral intake remained extremely poor. With her permission, a nasogastric tube was passed and a 4.2 MJ overnight feed was started. After 1 week her appetite had improved sufficiently, so that she began to eat the meals specially prepared for her by the ward-kitchen cook and she began to gain weight. This case illustrates the important role of psychiatric factors, not only in the classical cases of anorexia nervosa in the young, but also in the elderly. A combination of feeding techniques may tide the patient over the acute phase of illness and allow more rapid recovery.

\section{ARTIFICIAL NUTRITION}

\section{Nutrition teams}

Although oral and nasogastric tube feeding do not require the intervention of nutrition teams and can be carried out successfully by nursing, dietetic and medical staff on general wards, several studies have shown the importance of having a nutrition team to manage parenteral feeding and the more sophisticated methods of enteral tube feeding, such as percutaneous endoscopic gastrostomy (PEG; Allison, 1992; Lennard-Jones, 1992; Elia, 1993; Hull et al. 1993). Although there may be some advantage in having a full-time nutrition nurse with responsibilities throughout the hospital, the clinical, dietetic and pharmacist members of the team need only devote a portion of their time to this activity. Our own audit results confirm those of others, showing that having a nutrition team improves patient selection for treatment and greatly reduces complication rates and costs (Gallen et al. 1990). These considerations may more than outweigh the staff costs involved.

\section{Protocols and monitoring}

The nutrition team should develop its own written protocols for all procedures, such as insertion of central venous lines and PEG, and for the management and care of these subsequently. Procedures and all complications should be recorded in a standardized manner to allow regular auditing. There should also be serial data forms for recording serial changes in weight and biochemical and haematological results. It is only by attention to such details that the highest standards of care can be achieved.

\section{Indication, goals and aims}

It is not possible to be dogmatic about the timing of artificial nutrition, but as a general rule, and particularly where there is a history of previous weight loss, patients should not be allowed to starve for more than $7 \mathrm{~d}$ without some form of nutritional support. Twenty-five years ago the aims of nutritional support would have been described as achieving positive $\mathrm{N}$ balance and weight gain. Hyperalimentation regimens were intended to overwhelm the 
metabolic response to injury and illness with high energy and protein intakes. Studies have shown that such an approach can be counter-productive, causing complications such as fluid overload, accelerated $\mathrm{O}_{2}$ consumption and $\mathrm{CO}_{2}$ production resulting in respiratory failure, and fatty liver and increased fat synthesis without improvement in lean mass. As a result of such studies, we have adopted a more defensive role towards nutrition in the acute phase of illness, trying to minimize loss of tissue mass and preserve function using much lower levels of energy and protein intake than formerly. There are very few patients, for example, who benefit from more than 8-10 MJ (with non-protein energy divided equally between fat and carbohydrate) or $14 \mathrm{~g} \mathrm{~N}$ in the acute phase of illness. Taking differences in weight into account, the total energy requirement of patients in hospital varies from 1.1 to 1.5 times estimated energy requirements, with a $N$ requirement of $0.2-0.25 \mathrm{~g} / \mathrm{kg}$. During convalescence, when the patient is physically active and anabolism of lean mass is possible, higher intakes may sometimes be beneficial, not only in restoring function, but in gradually rebuilding lost tissue mass.

\section{Enteral nutrition}

Since the introduction of fine-bore nasogastric tubes in the 1970s and percutaneous endoscopic gastrostomy in the early 1980s, there has been a great increase in the use of enteral feeding in patients who are unwilling or unable to swallow, but in whom the gastrointestinal tract is intact. Gastrostomy is the management of choice in long-term enteral feeding, particularly in neurological dysphagia, where it has proved more effective and freer of complications than nasogastric feeding (Park et al. 1992). Our own 4-year audit of its use, mainly in patients with motor neurone or cerebrovascular disease (Hull et al. 1993), has shown the contribution that an expert nutrition team may make to a low complication rate. The use of careful protocols, an educational programme for the patient and the family on how to manage the feeding system at home, and regular follow-up were all important factors in its success. In a recent study (J. Rawlings, A. Gazis and S. P. Allison, unpublished results), we have found that, in an unselected group of patients presenting with motor neurone disease to the neurological department, about one-quarter were suitable for percutaneous endoscopic gastrostomy and benefited from it at some time during their illness. At best it prolonged good-quality life; at worst it provided useful palliation and a route of administration of fluids and drugs as well as nutrition.

Enteral feeding has been increasingly used in Intensive Care Units at the expense of parenteral feeding. Not only is it less expensive, but it may protect the immune and barrier role of the gut as well as the function of the hepatobiliary system. This technique may also have a transitional role in patients who are being weaned from parenteral nutrition.

\section{Parenteral nutrition}

This may be regarded as the management strategy for acute and chronic gastrointestinal failure in the same sense that dialysis is the management strategy for acute and chronic renal failure, or ventilation that of respiratory failure. The patient's physiology is maintained while the underlying pathology is being treated, or resolves naturally. Par excellence, parenteral nutrition requires an expert team to manage it successfully. Infective, mechanical and metabolic complications are much higher without such expert attention 
(Allison, 1992; Lennard-Jones, 1992; Elia, 1993). The technique was first introduced for administration via peripheral veins by Wretlind and his colleagues in Sweden in the 1950s and $60 \mathrm{~s}$. With the introduction of improved cannulas, there has been a swing back to the use of this technique, at least for short periods of parenteral nutrition lasting up to 3 weeks. The introduction by Dudrick et al. (1968) of feeding by the central venous route has allowed the use of hyperosmolar feeds. In a 10-year retrospective audit (Shields et al. 1996), we have assessed the outcome and complication rate from this technique in patients undergoing a period of parenteral nutrition in hospital while recovering from gastrointestinal failure. In a non-malignant group fed for an average of $50 \mathrm{~d}$, there was a high recovery rate and a 75\% survival 10 years later. Because of their prolonged period of gastrointestinal failure, it could be argued that these patients would not have survived without this treatment, and that, with such an outcome, the cost of treatment was justified. The cost per year of life saved was in fact $£ 4700$, which is comparable with other wellaccepted technologies, such as renal replacement therapy. O'Hanrahan \& Irving (1992) have also shown the high survival rate and good quality of life of patients undergoing parenteral nutrition at home for chronic gastrointestinal failure. Such studies form the basis for arguing the case with hospital managers for appointing a nutrition team as part of the overall strategy for nutritional care in the hospital.

\section{SUMMARY AND CONCLUSIONS}

Treatable malnutrition may occur in up to $25 \%$ of hospital patients, depending on the speciality concerned. Nutritional status may also deteriorate during a prolonged hospital stay. The management and prevention of malnutrition requires a climate in which hospital managers have a positive policy towards nutritional care, a screening system to identify the patients at risk, and appropriate protocols for action. Catering services need to be reorganized to address the problems of the sick, so that appropriate food is not only prepared but delivered in a way which makes it likely to be consumed. For the optimal management of artificial nutrition by the enteral or parenteral route, a skilled nutrition team is both necessary and cost-effective.

\section{REFERENCES}

Allison, S. P. (1992). The uses and limitations of nutritional support. Clinical Nutrition 11, 319-330.

Arora, N. S. \& Rochester, D. F. (1982). Respiratory muscle strength and maximal voluntary ventilation in undernourished patients. American Review of Respiratory Disease 126, 5-8.

Bastow, M. D., Rawlings, J. \& Allison, S. P. (1983). Benefits of supplementary tube feeding after fractured neck of femur: a randomised controlled trial. British Medical Journal 287, 1589-1592.

Bastow, M. D., Rawlings, J. \& Allison, S. P. (1985). Overnight nasogastric tube feeding. Clinical Nutrition 4, 7-11.

Brozek, J. (1990). Effects of generalised malnutrition on personality. Nutrition 6, 389-395.

Chandra, R. K. (1988). Immunity and infection. In Nutrition and Metabolism in Patient Care, pp. 598-604 [J. M. Kinney, K. N. Jeejeebhoy, G. L. Hill and O. E. Owen, editors]. Philadelphia, PA: W. B. Saunders.

Delmi, M., Rapin, C.-H., Bengoa, J. M., Delmas, P. D., Vasey, H. \& Bonjour, J. P. (1990). Dietary supplementation in elderly patients with fractured neck of femur. Lancet 335, 1013-1016.

Department of Health and Nutrition Task Force Hospital Catering Project Team (1995). The Health of the Nation. Nutrition Guidelines for Hospital Catering. Department of Health Document G05/002 3120. London: H.M. Stationery Office.

Dudrick, S. J., Wilmore, D. W., Vars, H. M. \& Rhoades, J. E. (1968). Long term total parenteral nutrition with growth, development and positive nitrogen balance. Surgery 64, 134-141. 
Elia, M. (1993). Artificial nutritional support in clinical practice in Britain. Journal of the Royal College of Physicians, London 27, 8-15.

Gallen, I., Ispahani, P., Kendall, J. \& Allison, S. P. (1990). Increased use of specialized TPN ward reduces both infection rate and cost of TPN. Clinical Nutrition 9, Special Suppl., 64.

Hill, G. L. (1992). Disorders of Nutrition and Metabolism in Clinical Surgery. Edinburgh: Churchill Livingstone.

Hull, M. A., Rawlings, J., Murray, F. E., Field, J., McIntyre, A. S., Mahida, Y. R., Hawkey, C. J. \& Allison, S. P. (1993). An audit of outcome of long-term enteral nutrition using percutaneous endoscopic gastrostomy (PEG). Lancet 340, 869-872.

Larsson, J., Unosson, M., Ek, A.-C., Nilsson, L., Thorslund, S. \& Bjurulf, P. (1990). Effect of dietary supplement on nutritional status and clinical outcome on 501 geriatric patients - a randomized study. Clinical Nutrition 9, 179-184.

Lennard-Jones, J. E. (editor) (1992). In A Positive Approach to Nutrition as Treatment. London: King's Fund Centre.

Lennard-Jones, J. E., Arrowsmith, H., Davison, C., Denham, A. F. \& Micklewright, A. (1995). Screening by nurses and junior doctors to detect malnutrition when patients are first assessed in hospital. Clinical Nutrition 14, 336-340.

McWhirter, J. P. \& Pennington, C. R. (1994). Incidence and recognition of malnutrition in hospital. British Medical Journal 308, 945-948.

Mansell, P. I., Fellows, I. W., Macdonald, I. A. \& Allison, S. P. (1990). Defect in thermoregulation in malnutrition reversed by weight gain. Physiological mechanisms and clinical importance. Quarterly Journal of Medicine 280, 817-829.

Ödlund Olin, A., Osterberg, P. \& Hadell, K. (1994). Energy-enriched hospital food to improve energy intake. Journal of Parenteral and Enteral Nutrition 18, Suppl., $31 \mathrm{~S}$.

O'Hanrahan, T. \& Irving, M. H. (1992). The role of home parenteral nutrition in the management of intestinal failure - Report of 400 cases. Clinical Nutrition 11, 331-336.

Park, R. H. R., Allison, M. C., Lang, J., Spence, E., Morris, A. J., Danesh, B. J. Z., Russell, R. I. \& Mills, P. R. (1992). Randomised comparison of percutaneous endoscopic gastrostomy and nasogastric tube feeding in patients with persisting neurological dysphagia. British Medical Journal 304, 1406-1409.

Rana, S. K., Bray, J., Menzies-Gow, N., Jameson, J., Payne-James, J. J., Frost, P. \& Silk, D. B. A. (1992). Short term benefits of postoperative oral dietary supplements in surgical patients. Clinical Nutrition 11, 337-344.

Reilly, H. M. (1996). Screening for nutritional risk. Proceedings of the Nutrition Society 55, 841-853.

Reilly, J. J., Hull, S. F., Albert, N., Waller, A. \& Bringardener, S. (1988). Economic impact of malnutrition: a model system for hospitalised patients. Journal of Parenteral and Enteral Nutrition 12, 371-376.

Robinson, G., Goldstein, M. \& Levine, G. M. (1987). Impact of nutritional status on DRG length of stay. Journal of Parenteral and Enteral Nutrition 11, 49-51.

Russell, D. M., Leiter, L. A., Whitwell, J., Marliss, E. B. \& Jeejeebhoy, K. N. (1983). Skeletal muscle function during hypocaloric dieting and fasting: a comparison with standard nutritional assessment parameters. American Journal of Clinical Nutrition 37, 133-138.

Shields, P. L., Field, J., Rawlings, J., Kendall, J. \& Allison, S. P. (1996). Long-term outcome and costeffectiveness of parenteral nutrition for acute gastrointestinal failure. Clinical Nutrition 15 (In the Press).

Veterans' Affairs Total Parenteral Nutrition Study Group (1991). Perioperative total parenteral nutrition in surgical patients. New England Journal of Medicine 325, 525-532.

Von Meyenfeldt, M. F., Meijerink, W. J. H. J., Rouflart, M. M. J., Buil-Maassen, M. T. H. J. \& Soeters, P. B. (1992). Perioperative nutritional support: a randomized clinical trial. Clinical Nutrition 11, 180-186.

Windsor, J. A., Knight, G. S. \& Hill, G. L. (1988). Wound healing response in surgical patients: recent food intake is more important than nutritional status. British Joumal of Surgery 75, 135-137.

Winick, M. (editor) (1979). Hunger Disease: Studies by the Jewish Physicians in the Warsaw Ghetto. New York: Wiley. 